\title{
Maternally derived glucocorticoid inhibits adrenal development in offspring induced by prenatal dexamethasone exposure via IGF1
}

\author{
Yawen Chen ${ }^{1}$, Xuan Xia ${ }^{1}$, Guanghui Chen ${ }^{1}$, Jiangang $\mathrm{Cao}^{1}$, and Hui Wang ${ }^{1}$ \\ ${ }^{1}$ Wuhan University School of Basic Medical Sciences
}

October 6, 2020

\begin{abstract}
Background and Purpose: Adverse environments during pregnancy can increase susceptibility to chronic diseases in adult offspring, which might be related to intrauterine glucocorticoid-induced multi-organ developmental programming and homeostasis alterations. Dexamethasone is widely used for preterm delivery-related pregnancy diseases. Previous studies suggested that prenatal dexamethasone exposure (PDE) could cause developmental toxicity of adrenal gland in offspring and the underlying mechanism has not been elucidated. Experimental Approach: Wistar rats were subcutaneously injected with dexamethasone $(0.2 \mathrm{mg} / \mathrm{kg} \cdot \mathrm{d})$ during gestational day 9-20. The placentas and serum and adrenal samples were collected to identify the related indicators. In vitro, human adrenocortical cell lines (NCI-H295R) were treated with cortisol and dexamethasone to confirm the molecular mechanism, respectively. Key Results: PDE caused a low level of maternally derived glucocorticoid in male fetal blood. Furthermore, the serum corticosterone level, the H3K27ac and expression levels of the adrenal insulin-like growth factor 1 (IGF1), and steroidogenic function continuously decreased in the PDE male offspring rats. With chronic stress, the serum corticosterone level increased in the adult PDE offspring, while the above indicators were also increased correspondingly. In vitro, we further confirmed that the endogenous glucocorticoid positively programmed the adrenal IGF1 expression and steroidogenesis through the GR $\alpha / \mathrm{miR}-370-3 \mathrm{p} /$ Sirt3 pathway. Conclusion and Implications: The low level of maternally derived glucocorticoid induced by PDE caused adrenal insufficiency of adult offspring rats through epigenetic positive programming of the glucocorticoid (GC)-IGF1 axis. This study firstly confirmed that exogenous glucocorticoids (dexamethasone) can alter the adrenal development programming and homeostasis in offspring by inhibiting maternal adrenal function.
\end{abstract}

\section{Introduction}

In the 1990s, the British scholar Barker firstly proposed the hypothesis of increased incidence of metabolic syndrome in adults with low birth weight and the developmental origin of adult diseases based on the results of a large-scale epidemiological survey. Over the past three decades, scholars from many countries have carried out evidence-based research on prenatal adverse environment, fetal birth weight, and adult chronic diseases, and put forward a new concept of the origin of human diseases called the Developmental Origins of Health and Disease (DOHaD). To date, fetal development programming has become an essential theory for understanding healthy development programming throughout the life cycle (Fleming et al. , 2018). Dexamethasone is one of synthetic glucocorticoids that can promote fetal lung maturation, reduce the occurrence of neonatal respiratory distress syndrome and perinatal mortality, so it is widely used for preterm delivery and related pregnancy diseases (Porto et al. , 2011; Crowther et al. , 2015). The World Health Organization (WHO) reported that the prevalence of prenatal synthetic glucocorticoid treatment for premature labor at 22-36 weeks of pregnancy is $54 \%$ in the maternal and child health survey data of 359 institutions in 29 countries and is as high as $91 \%$ in some countries (Vogel et al. , 2014). However, the use of prenatal dexamethasone is a double-edged sword. A large number of studies have confirmed that repeated dexamethasone treatment has fetal developmental toxicity, which can cause low birth weight, multiple organ development abnormalities, and susceptibility to chronic diseases in adulthood (Asztalos et 
al. , 2010; Elfayomy et al. , 2014).

As the terminal effector of the hypothalamic-pituitary-adrenocortical (HPA) axis, the adrenal gland is the organ with the earliest and fastest growth of the HPA axis in utero. The adrenal gland is the organ of glucocorticoid synthesis and secretion. Accumulating studies suggest that basic glucocorticoid levels in utero play an important role in fetal maturity and postnatal fate (Moisiadis et al. , 2014a), and its alteration caused by adverse environments during pregnancy can lead to change in insulin-like growth factor 1 (IGF1) level in fetal blood (Fowden, 2003), catch-up growth of the IUGR progeny and the occurrence of metabolic diseases (Mericq et al. , 2017). Therefore, the development of the adrenal gland is the key to determine the postnatal fate. IGF1 expression in the adrenal gland begins in the second trimester of pregnancy, occurring only later than in the liver, and IGF1 can regulate adrenal development and functional differentiation (Han et al. , 1988). IGF1 and IGF1R are widely expressed in the adrenal cortex, upregulating steroid synthase through phosphorylation of the PI3K/Akt pathway to promote steroidogenesis (Raha et al. , 2007; Sirianni et al. , 2007). Our previous research found that prenatal dexamethasone exposure (PDE) could cause adrenal dysplasia in offspring mice, accompanied by decreased serum corticosterone levels in maternal and fetal mice (Xu et al. , 2011). However, is this adrenal developmental dysfunction in offspring associated with changes in maternal adrenal function? Is IGF1 involved in PDE-induced programming alteration of adrenal development in offspring? We have not seen relevant reports so far.

"Intrauterine programming" is the process of permanent alterations in fetal tissue morphology and function caused by intrauterine environment changes. And it is also the pathophysiological basis for the occurrence of fetal-originated adult diseases (Moisiadis et al. , 2014a). Epigenetics can lead to heritable changes in gene function that do not involve changes in the DNA sequence, including DNA methylation, histone modification, and non-coding RNA (such as miRNAs). Numerous studies have suggested that glucocorticoid-induced intrauterine programming alterations are related to abnormal expression of some critical genes regulated by miRNAs (Clayton et al. , 2018), and miRNAs can regulate histone acetylation and expression levels of downstream target genes by targeting histone deacetylase (Gao et al. , 2018; Xing et al. , 2019). Our recent studies have confirmed that abnormal histone acetylation modification was involved in multi-organ development programming and related disease susceptibility caused by PDE (Liu et al. , 2018; Xiao et al. , 2018; Li et al. , 2019). Therefore, we speculate that low levels of maternally derived glucocorticoid caused by PDE may regulate IGF1 histone acetylation and expression through miRNAs in fetal adrenal, thereby resulting in its abnormal function development in offspring.

In this study, based on a stable PDE rat model established in our laboratory, we firstly confirmed the phenomenon of the low level of maternally derived glucocorticoid in fetal blood. Then, a programming alteration of GC-IGF1 axis was explored by detecting blood corticosterone, adrenal IGF1 expression and steroidogenesis function before and after birth, as well as its effects after chronic stress in adults. Finally, the histone acetylation regulating mechanism of glucocorticoid-dependent IGF1 expression was elucidated in combination with human adrenocortical cells in vitro. This study will elucidate the programming effect of maternal glucocorticoid for offspring adrenal development programming and homeostasis alterations, and provide an essential theoretical and experimental basis for explaining the intrauterine development origin of PDE-induced multi-organ dysplasia and related disease occurrence and development in adults.

\section{Materials and methods}

\subsection{Animals and treatment}

Specific pathogen-free adult Wistar female (mean body weight: $209 \pm 12 \mathrm{~g}$ ) and male (mean body weight: $258 \pm 17 \mathrm{~g}$ ) rats (certification No. 42000600002258, license No. SCXK (E) 2012-2014) were provided by the Experimental Centre of the Hubei Medical Scientific Academy (Wuhan, China). Animal experiments were performed in the Center for Animal Experiment of Wuhan University (Wuhan, China), which has been accredited by the Association for Assessment and Accreditation of Laboratory Animal Care International. The Committee on the Ethics of Animal Experiments of the Wuhan University School of Medicine approved the protocol (permit number: 14016). All animal experimental procedures were performed following the 
Guidelines for the Care and Use of Laboratory Animals of the Chinese Animal Welfare Committee.

Animals were housed in metal cages in an air-conditioned room under standard conditions (environmental temperature: $18-22^{\circ} \mathrm{C}$; humidity: $40-60 \%$; light cycle: $12 \mathrm{~h}$ light-dark cycle; $10-15$ air changes per hour) and allowed free access to rat chow and tap water. All rats were acclimated one week before experimentation, and two female rats were placed together with one male rat overnight in a cage for mating. Mating was confirmed by the presence of sperm in the morning vaginal smear, recorded as the gestational day (GD) 0. Pregnant rats were transferred to individual cages and then randomly divided into the control group and PDE group. From GD 9 to GD 20, the PDE group was subcutaneously administrated with $0.2 \mathrm{mg} / \mathrm{kg} \cdot \mathrm{d}$ dexamethasone (Shuanghe, Pharmaceutical Co., Wuhan, China), while the control group was administered an equal volume of saline every morning (between 08:00-09:00 a.m.).

On GD 20, a subgroup of pregnant rats was anesthetized by isoflurane (Baxter Healthcare Co., Deerfield, IL, USA; 10019-360-40) inhalation, and the fetuses were removed by cesarean section. Each fetoplacental unit was quickly removed from the uterus, and the fetuses were weighed after being dried on filter papers. Pregnant rats with litter sizes of 12 to 14 were considered qualified. The IUGR diagnosis was based on two standard deviations of the fetal rat body weight lower than the mean weight of the control group. Fetal blood was pooled by female grouping per dam and was centrifuged for storage. The placental and fetal adrenal samples collected from littermates were pooled, immediately frozen in liquid nitrogen, and stored at -80degC for subsequent analyses. It should be noted that three whole fetal adrenals in three different fetal rats from each litter were randomly counted as one sample, and processed for gene analysis. The adrenals of five male fetuses in each group were randomly selected and fixed in $4 \%$ paraformaldehyde for $24 \mathrm{~h}$. They were then dehydrated with alcohol and embedded in paraffin for further analysis.

The remaining pregnant rats ( $\mathrm{n}=8$ for each group) underwent normal delivery. On postnatal day (PD) 1 , the numbers of pups were normalized to 8 pups per litter to assure adequate and standardized nutrition until weaning postnatal week (PW) 4. Three male offspring rats from each litter were selected and assigned to the studies at different postnatal time points or conditions (PW12, PW12 with chronic stress and PW28), respectively. The chronic stress test was achieved by forced 5-min ice-water swimming per day (5-7degC) from PW10 to PW12 [25]. At different postnatal time points, male rats were anesthetized with $2 \%$ isoflurane and euthanized. Then, the blood sample from the carotid artery was collected, the serum was isolated, and the adrenals and epididymis tissues were rapidly collected. All adrenals were dissected. The left partial adrenal was fixed in a $4 \%$ paraformaldehyde solution for histological examination, and the rest were separated and immediately frozen in liquid nitrogen, followed by storage at -80 for subsequent analyses. The schedule of animal treatment is shown in Fig S1.

\subsection{Cell culture and treatment}

The human adrenal cortical adenocarcinoma cell line (NCI-H295R) were plated into cell culture flasks with DMEM medium with $10 \%$ fetal bovine serum, $1 \%$ streptomycin, and penicillin in a $5 \% \mathrm{CO}_{2}$ humidified incubator at $37 \mathrm{degC}$. Cells were treated with dexamethasone $(0,20,100,500 \mathrm{nM})$ or cortisol $(300,150,75$ $\mathrm{nM}$ ) for $24 \mathrm{~h}$, and then harvested for further analysis. Chemically synthesized GR $\alpha$ plasmid, miR-370-3p mimics, Sirt3 siRNA oligo was obtained from Suzhou GenePharma Co., Ltd. (Suzhou, China). IGF1 (791MG) was purchased from Abcam. NCI-H295R cells were seeded into six-well plates at $1 \times 10^{5}$ cells/ well one day before GR $\alpha$ plasmid, miR-370-3p mimics, Sirt3 siRNA oligo transfection to reach the $50 \%$ confluency, respectively. After the transfection procedures were performed using Lipofectamine 3000 (Invitrogen, Thermo Fisher Scientific; L3000150) for $24 \mathrm{~h}$ according to the manufacturer's instructions, the transfection mixture medium was replaced by the medium with or without cortisol $(300 \mathrm{nM})$ or dexamethasone $(500 \mathrm{nM})$ for $24 \mathrm{~h}$. The cells were then lysed and total RNA was extracted using the TRIzol reagent (Invitrogen Co.; 15596018), or the cells were lysed using RIPA buffer for protein extraction.

\subsection{Serum corticosterone detection}

The concentration of serum corticosterone was measured by ELISA assay kits (R\&D Systems, Inc., Minneapolis, MN, USA) according to the manufacturer's protocol. The limit of detection for corticosterone 
concentration was $0.39 \mathrm{ng} / \mathrm{ml}$. The intra-assay and inter-assay coefficients of variation for corticosterone were 5.0 and $7.2 \%$, respectively.

\subsection{Luciferase reporter assay}

The Segment of 3'-UTR encompassing the miR-370-3p binding sites was amplified from the Sirt3 gene as the wild type (WT), correspondingly, a mutant 3'-UTR sequence was obtained using a mature technology, site-specific in vitro mutation. WT or mutant 3'-UTR was subsequently inserted downstream of the luciferase gene in the pmirGLO-control vector (Promega, USA). The NCI-H295R cells were seeded onto 96 well plates. After culturing for $24 \mathrm{~h}$, co-transfection of miR-370-3p vector and negative control (NC) along with a pmirGLO-control vector carrying WT or mutant 3'-UTR of Sirt3 into cells were performed using Lipofectamine 3000 reagent (Invitrogen, USA) according to the manufacturer's instructions. Then luminescence signal was recorded using the Dual-Luciferase Assay kit (Promega, USA) as indicated in the instructions. All assays were performed in triplicate in a single experiment and repeated for six times.

\subsection{Total RNA extraction and gene expression assay}

Total RNA extraction from the placental, adrenal, and NCI-H295R cells was performed using TRIzol reagent (Invitrogen Co.; 15596018) and following the manufacturer's protocol. The concentration and purity of the isolated RNA were determined by a spectrophotometer (NanoDrop 2000C, Thermo), and the RNA concentration was adjusted to $1 \mu \mathrm{g} / \mu \mathrm{l}$. Complementary DNA was synthesized from $1 \mu \mathrm{g}$ of total RNA according to the kit protocol and was stored at $-20^{\circ} \mathrm{C}$ for testing. RNA preparations were reverse transcribed to generate cDNA using the Reverse transcription reagent kit (Takara Biotechnology Co., Dalian, China) and miScript Reverse transcription kit (RiboBio, Guangzhou, China), respectively. Quantitative measurements of mRNA and miR expression were done using a SYBRGreen PCR kit (Applied Biosystems, ABI, 4472908) and miDETECT A Track miRNA quantitative real-time polymerase chain reaction (qRT-PCR) kit (RiboBio, Guangzhou, China) according to the manufacturer's guidelines and a StepOnePlus Real-time PCR system (Applied Biosystems, Foster City, CA, USA), using specific primers. U6 snRNA was used as an internal control for the miRNAs, GAPDH was used as an internal control for the adrenal samples, and calculated by the cycle threshold $2^{\mathrm{t}}$ method. The sequences of primers used in this experiment are shown in Table S1. All primers were synthesized by Sangon Biotech Co., Ltd. (Shanghai, China).

\subsection{Chromatin immunoprecipitation (ChIP) assay}

Homogenates of NCI-H295R cells and fetal adrenal were sheared using digestion/nuclei permeabilization buffer containing MNase at $37 \mathrm{degC} .1 \%$ formaldehyde was added directly to the homogenate of testicular tissue or scraped cells for $15 \mathrm{~min}$ at $37 \mathrm{degC}$ to cross-link DNA and its associated proteins. Glycine $(0.125 \mathrm{M}$ final concentration) was added to terminate the reaction for $8 \mathrm{~min}$. The lysates were then sonicated to shear the DNA to a size of 200-800 bp. After sonication, the samples were collected by centrifugation and were diluted with ChIP dilution buffer. The supernatant was used for each ChIP reaction, supplemented with ChIP dilution buffer and anti H3K27(1:50 dilution, ABclonal, A7253) or goat anti-rabbit IgG (1:50 dilution, ABclonal, AC005). Then, the DNA-protein-antibody complexes were captured with BSA-treated protein G agarose beads (EMD Millipore, Billerica, MA, USA). After incubation for $6 \mathrm{~h}$, beads were washed, and DNA-protein-antibody complexes were eluted, proteins were digested with proteinase K (EO0491, Beyotime Biotechnology, Nanjing, China) and ChIP-DNA was eluted using a DNA purification kits (Tiangen Biotech Co., Beijing, China). DNA from each input sample was extracted in parallel. For quantification by qPCR, ChIP-DNA was diluted and run using the SYBR Green PCR kit with specific primers listed in TableS2. All primers were synthesized by Sangon Biotech Co., Ltd. (Shanghai, China).

\subsection{Total protein extract and western blotting assay}

Briefly, placental, adrenal tissue, and NCI-H295R cells were rinsed three times with ice-cold phosphatebuffered saline (PBS) and then lysed for $30 \mathrm{~min}$ at $4 \operatorname{deg} \mathrm{C}$ in radioimmunoprecipitation assay (RIPA) lysis buffer containing phosphatase inhibitor cocktail, followed by the BCA Assay kit for protein quantification. A total of $30 \mu \mathrm{g}$ of proteins was loaded to each lane, isolated by $10 \%$ sodium dodecyl sulfate-polyacrylamide 
gel electrophoresis (SDS-PAGE). For the transfer to the membrane, a wet transfer was performed using an assembly of polyvinyl diisopropyl fluoride (PVDF) membranes (Millipore, MA, USA). The transfer was performed at 100v, $350 \mathrm{mAmp}$ for $1 \mathrm{~h}$. After three washes with TBST $(25 \mathrm{mM}$ Tris-HCl, $50 \mathrm{mM} \mathrm{NaCl}$, $0.05 \%$ Tween-20), the membrane was blocked with $5 \%$ skim milk in TBST for $1 \mathrm{~h}$. The primary antibodies, including anti-P Glycoprotein (P-gp) (1:1000 dilution, Abcam, ab170904) and anti-hydroxysteroid 11betadehydrogenase 1, (11ß-HSD1) (1:1000 dilution, Abcam, ab39364), anti-11ß-HSD2 (1:1000 dilution, Santa Cruz, sc-365529), anti-Sirt3 (1:1000 dilution, ABclonal, A7307), anti-IGF1 (1:200 dilution, Santa Cruz, sc9013) and anti-steroidogenic acute regulatory protein (StAR) (1:1000 dilution, ABclonal, A16432) were added to TBST in 5\% skim milk and placed on constant shaking at $4^{\circ} \mathrm{C}$ overnight. After incubation and washing, the membranes were incubated with secondary antibodies (anti-mouse and anti-rabbit conjugated with horseradish peroxidase) at a 1:5000 dilution for $1 \mathrm{~h}$ and were detected using an enhanced chemiluminescent detection kit (Bridgen, Beijing, China; D046). Densitometric analyses were performed using ImageJ software (National Institutes of Health).

\subsection{Immunohistochemical assays}

Immunohistochemistry analysis was performed by using a DAB staining kit (Gene Tech Co. Ltd., Shanghai, China; GK347011) to determine the expression levels of StAR (1:1000 dilution) in adrenals at GD20, PW12, and PW28. Adrenals were cut into $5 \mu \mathrm{m}$-thick slices along the longitudinal axis. For immunohistochemical staining, the slices were dewaxed and washed in PBS. After antigen retrieval, adrenal sections were blocked in 5\% blocking serum at room temperature for $30 \mathrm{~min}$ and probed with primary antibodies overnight at 4. After primary antibody incubation, sections were incubated with a biotinylated secondary antibody and then with an avidin-biotinylated horseradish peroxidase complex solution according to the manufacturer's directions. Finally, peroxidase activity was determined with a DAB staining kit (Gene Tech Company, Ltd., Shanghai, China). All the images were analyzed using Image-Pro Plus software (version 6.1, Media Cybernetics, Silver Spring, USA). The intensity of StAR staining was determined by measuring the mean optical density in five random fields for each sample, and five sections from different samples per group were used for quantification.

\subsection{Statistical analysis}

SPSS 19 (SPSS Science Inc., Chicago, Illinois) and Prism 6.0 (Graph Pad Software, La Jolla, CA, USA) were used to perform data analysis. Quantitative data expressed as the mean \pm S.E.M. Two-tailed Student'st -test was used for comparisons between the control and treated groups, and studies involving more than two groups, data were evaluated with one-way Analysis of Variance (ANOVA) followed by the Tukey's post hoc test. Statistical significance was defined as $P<0.05$.

\section{Results}

3.1 The phenomenon of low maternally derived glucocorticoid exposure in fetal blood induced by PDE and its occurrence mechanism

The level of basic glucocorticoid (cortisol in humans and corticosterone in rodents) during the intrauterine period is a key factor in regulating the maturity and function of fetal tissue. To explore the effects of PDE on fetal serum glucocorticoid levels and its occurrence mechanism, we first examined the corticosterone levels in maternal and fetal serum. Compared with the maternal control rats, the serum corticosterone levels were significantly reduced in the PDE group $(P<0.05$, Fig $1 \mathrm{~A})$, while the serum ACTH levels were not markedly changed (Fig 1B). Meanwhile, qRT-PCR analysis showed that the mRNA expressions of adrenal steroidogenic genes, including StAR, P450scc, P450c21, and P450c11, were significantly reduced in the PDE maternal rats $(P<0.05, P<0.01$, Fig 1C). Compared with the fetal control rats, the serum corticosterone levels were also significantly reduced in the PDE group $(P<0.01$, Fig 1D). The placental glucocorticoid barrier is a crucial factor controlling the transfer of maternal glucocorticoid to the fetus. Hence, we examined the expression of the placental glucocorticoid barrier-related transport protein (P-gp) and glucocorticoid metabolic enzymes (11ß-HSDs). The results showed that compared with the control group, the mRNA expression of P-gp encoding genes - Mdr1a and Mdr1b in placental were significantly reduced in the PDE group, and the 
mRNA expression of placental 11ß-HSD1 was increased $(P<0.01$, Fig $1 \mathrm{E})$ while that of $11 \beta$-HSD2 was not

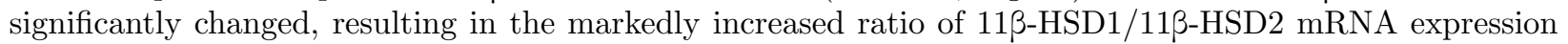
$(P<0.01$, Fig $1 \mathrm{~F})$. Meanwhile, western blotting analysis also suggested similar conclusions at the protein expression level $(P<0.05, P<0.01$, Fig $1 \mathrm{G}-\mathrm{I})$. Furthermore, we analyzed the correlation between maternal serum corticosterone levels and fetal serum corticosterone levels, and found a significant positive correlation between them $(P<0.01$, Fig $1 \mathrm{~J})$. These results indicated that the serum corticosterone levels were reduced in the PDE fetal rats, and its occurrence was mainly related to the decrease of maternal corticosterone levels and the open placental glucocorticoid barrier.

\subsection{Changes in fetal adrenal GC-IGF1 axis andsteroidogenic function induced by PDE and its possible mechanisms}

Compared with the control group, the mRNA expressions of fetal adrenal steroidogenic genes, including StAR, P450scc, P450c21, and P450c11, were reduced in the PDE group $(P<0.01$, Fig 2A), and StAR protein expression was also significantly reduced $(P<0.05$, Fig $2 \mathrm{~B}, \mathrm{C})$. We also observed the expression of fetal adrenal IGF1 and its downstream signaling pathway, and found that the mRNA expression levels of IGF1, IGF1R, and Akt1 were markedly decreased in the PDE group $(P<0.01$, Fig 2D). Further, we explored their occurrence mechanism. The results showed that compared with the control, adrenal glucocorticoid receptor $\alpha(\mathrm{GR} \alpha)$ mRNA expression was significantly increased in the PDE fetal rats $(P<0.01$, Fig $2 \mathrm{E})$. Fetal adrenal microarray analysis indicated that the expression of miR-370-3p was reduced in the PDE group (Fig 2F), and Sirt3 might be the target gene of miR-370-3p through bioinformatics prediction. We confirmed that adrenal miR-370-3p expression was decreased and Sirt3 expression increased in the PDE fetal rats using qRT-PCR $(P<0.01$, Fig $2 \mathrm{G}, \mathrm{H})$. Moreover, screening revealed that the H3K9ac and H3K27ac levels of the IGF1 promoter region were reduced in the PDE fetal adrenal glands $(P<0.01$, Fig $2 \mathrm{I})$ while the H3K14ac level was not significantly changed (Fig 2I). These data indicated that the GR $\alpha /$ miR-3703p/Sirt3/IGF1 signaling pathway might mediate the adrenal low steroidogenic function in the PDE fetal rats through histone acetylation modification.

3.3 Changes in adult adrenal GC-IGF1 axis and steroidogenic function induced by PDE and its possible mechanisms

To confirm that the PDE-induced inhibition of fetal adrenal steroidogenesis could continue after birth and explore its epigenetic programming mechanism, we detected the changes in adrenal steroidogenesis in PW 12 and PW28. The results showed that compared with the respective controls in PW12 and PW28, the serum corticosterone levels and the mRNA expression of the adrenal steroidogenic genes (StAR and P450scc) and IGF1 signaling pathway were significantly reduced in the PDE group $(P<0.05, P<0.01$, Fig $3 \mathrm{~A}$, B and D), and immunohistochemical examination confirmed that the protein expression of StAR was also significantly reduced $(P<0.05, P<0.01$, Fig 3C). Meanwhile, PDE decreased the expression of adrenal GR $\alpha$ and miR370-3p, increased Sirt3 expression, and decreased the H3K27ac level of IGF1 promoter region $(P<0.05, P$ $<0.01$, Fig 3E-H), while H3K9ac and H3K14ac levels of IGF1 did not change significantly (Fig 3H). This suggested that the adrenal steroidogenic function was continually reduced in the PDE offspring after birth, and GR $\alpha /$ miR-370-3p/Sirt3/IGF1 signaling pathway might mediate the low adrenal steroidogenesis in the PDE adult offspring rats through histone acetylation modification.

3.4 Changes in positive programming of adrenal GC-IGF1 axis in PDE adult offspring with chronic stress and its related mechanisms

Furthermore, we confirmed the changes in positive programming of the adrenal GC-IGF1 axis in PDE adult rats through chronic stress (2 weeks of ice-water swimming). We found that serum corticosterone levels increased in both the control group and the PDE group with chronic stress, but the increase was more significant in the PDE group $(P<0.05, P<0.01$, Fig 4A). Next, compared with the groups without chronic stress, the expressions of adrenal IGF1 and steroidogenesis-related genes (StAR and P450scc) were significantly decreased in the control group with chronic stress, while significantly increased in the PDE group with chronic stress $(P<0.05, P<0.01$, Fig 4B, C). These results indicated that the adrenal GC-IGF1 
axis of the normal offspring rats showed the negative changes, while that of the PDE offspring rats showed positive changes.

We also detected the changes of relevant indicators of the GC-IGF1 axis positive programming mechanism in the PDE offspring rats. Compared with the respective groups without chronic stress, the expression of adrenal GR $\alpha$ was increased, the expression of miR-370-3p was decreased, the expression of Sirt3 was increased, and the H3K27ac level of IGF1 promoter region was decreased in the control group with chronic stress $(P<0.05, P<0.01$, Fig $4 \mathrm{D}-\mathrm{G})$. However, these indicators showed consistent opposite changes in the PDE group with chronic stress $(P<0.05, P<0.01$, Fig $4 \mathrm{D}-\mathrm{G})$. These data indicated that the expression changes of the GR $\alpha / \mathrm{miR}-370-3 \mathrm{p} /$ Sirt3 pathway and the H3K27ac level of the IGF1 promoter region were presented in a glucocorticoid-dependent manner in the adult PDE offspring rats.

\subsection{Inhibition of dexamethasone on IGF1 andsteroidogenic function inNCI-H295R cells}

Dexamethasone can easily enter the fetal blood through placentas. This study sought to determine whether dexamethasone in utero affected fetal adrenal steroidogenic function and exhibited an intrauterine programming effect. First, we detected the effects of dexamethasone with different concentrations $(0,20,100$, $500 \mathrm{nM}$ ) on the mRNA and protein expression of IGF1 and StAR, and the level of intracellular cortisol in the human adrenocortical cell line (NCI-H295R). The results showed that compared with the control, dexamethasone concentration-dependently decreased the above indicators $(P<0.05, P<0.01$, Fig 5A-D). Compared with the $500 \mathrm{nM}$ dexamethasone group, exogenous IGF1 treatment reversed the inhibitory effect of dexamethasone on StAR expression $(P<0.05, P<0.01$, Fig $5 \mathrm{E})$. Meanwhile, we observed the effects of dexamethasone on histone acetylation of the IGF1 promoter region, and found that the H3K9ac level of the IGF1 promoter region was significantly reduced $(P<0.05$, Fig $5 \mathrm{~F})$, while there were no significant changes in the H3K14ac and H3K27ac levels (Fig $5 \mathrm{~F}$ ). This suggested that dexamethasone reduced steroidogenic function by inhibiting the H3K9ac and expression levels of IGF1 in the human adrenocortical cells.

3.6 Inhibition of GC-IGF1 axis andsteroidogenic function by low physiological concentrations of cortisol in NCI-H295R cells

As mentioned above, we had confirmed a low level of maternal glucocorticoid in fetal rats induced by PDE. Here, we further explored the effect of low glucocorticoid levels on adrenal steroidogenic function in NCI-H295R cells. Studies have reported that physiological cortisol concentration fluctuation range of normal neonatal is $11.67+/-4.68 \mu \mathrm{g} / \mathrm{dl}(303.5+/-129.1 \mathrm{nM})$, while neonates treated with dexamethasone during pregnancy have a range of $8.45+/-6.31 \mu \mathrm{g} / \mathrm{dl}(233.1+/-174.1 \mathrm{nM})$ (Srivastava et al. , 1994). Thus, we treated NCI-H295R cells with different concentrations (300, 150, and $75 \mathrm{nM}$ ) of cortisol for $24 \mathrm{~h}$. Compared with the physiological control (300 nM) (Ruyet al. , 2017), the StAR expression and intracellular cortisol level were decreased in a cortisol concentration-dependent manner $(P<0.05, P<0.01$, Fig 6A,B). Meanwhile, the expression of GR $\alpha$ and miR-370-3p were decreased, the expression of Sirt3 was increased, and the H3K27ac and expression levels of IGF1 promoter region were decreased $(P<0.05, P<0.01$, Fig 6C-G), while the H3K9ac and H3K14ac levels of IGF1 promoter region did not change significantly (Fig 6F). Moreover, the protein expressions of StAR, Sirt3, and IGF1 were consistent with their mRNA expression (Fig $6 \mathrm{H})$. These data indicated that low physiological concentrations of cortisol might inhibit steroidogenic function by altering GR $\alpha / \mathrm{miR}-370-3 \mathrm{p} / \mathrm{Sirt3} / \mathrm{IGF} 1$ expression in the human adrenocortical cells.

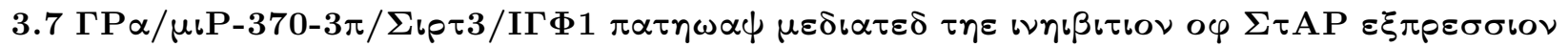

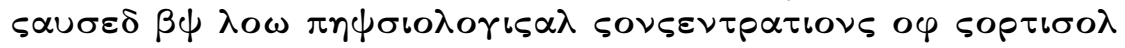

To further confirm that the GR $\alpha$ /miR-370-3p/Sirt3/IGF1 pathway mediated the inhibitory effect of low physiological concentrations of cortisol on StAR expression, we conducted a series of interference experiments. Firstly, we treated the NCI-H295R cells with exogenous IGF1, and found that IGF1 could significantly reverse the inhibitory effect of $75 \mathrm{nM}$ cortisol on StAR expression $(P<0.05, P<0.01$, Fig 7A). Secondly, we observed that $75 \mathrm{nM}$ cortisol decreased the H3K27ac and expression levels of the IGF1, while treatment with the Sirt3 siRNA or miR-370-3p mimics reversed the above effects of cortisol $(P<0.05, P<0.01$, Fig 7B, C, E, F). And miR-370-3p over-expression also significantly reversed the effect of $75 \mathrm{nM}$ cortisol on Sirt3 
expression $(P<0.05$, Fig 7D). Furthermore, we found that the 3'UTR of Sirt3 mRNA contains a potential miR-370-3p binding site via bioinformatics prediction, and successfully constructed the double luciferase expression vectors containing the Sirt3 wild type or mutant type (416-422 location) sequence (Fig 7G). The results showed that compared to the respective control, co-transfection of miR-370-3p mimics with Sirt3 WT 3'UTR luciferase reporter plasmids significantly reduced luciferase activity, while co-transfection of miR-370-3p mimics with Sirt3 MUT 3'UTR luciferase reporter plasmids showed no significant change in the luciferase activity $(P<0.05, P<0.01$, Fig $7 \mathrm{H})$. In addition, $75 \mathrm{nM}$ cortisol reduced the GR $\alpha$ expression, and transfection with GR $\alpha$ plasmid could significantly reverse the inhibitory effect of $75 \mathrm{nM}$ cortisol on miR-370$3 \mathrm{p}$ expression and the H3K27ac and expression levels of IGF1 $(P<0.01$, Fig 7I-K). These data indicated that low physiological concentrations of cortisol reduced GR $\alpha$ expression, downregulating miR-370-3p to target upregulation of Sirt3, which resulted in the decreased H3K27ac and expression levels of IGF1. Ultimately, the StAR expression was reduced.

\section{Discussion}

4.1 The basis of establishing a PDE rat model and the significance of offspring's abnormal adrenal function in the occurrence of fetal-derived diseases

Dexamethasone is often given to treat pregnant women at risk of premature delivery in the second and third trimester of pregnancy (Gilstrap et al. , 2001). The classic clinical application of dexamethasone in the treatment of premature infants is an intramuscular injection every $12 \mathrm{~h}, 6 \mathrm{mg}$ each time, for a total of 4 times. However, approximately $1 / 3$ of pregnant women change from preventive to continuous administration due to the difficulty in early diagnosis of preterm delivery and the inefficacy of a single course in some pregnant women, resulting in multiple courses of treatment, sometimes more than 11 courses (Quinlivan et al. , 1998; Murphy et al. , 2008). Accumulating animal experiments showed that multi-course treatment could cause a series of long-term adverse effects (Elfayomy et al. , 2014). The National Institutes of Health (NIH) suggested that more animal experiments were needed to explore the long-term effects of multi-course treatment.

In humans, the adrenal fetal zone and permanent zone gradually forms a transitional zone in the $16^{\text {th }}$ $20^{\text {th }}$ weeks during pregnancy, which has some typical characteristics of the adrenal cortex (Mesianoet al. , 1993). In rats, the development of the adrenal cortex starts from the migration of corpus callosum epithelial cells of the urogenital system from GD 9 (Hatanoet al. , 1996). The vital organs that sustain life have also developed and have corresponding functions in the second and third trimesters of pregnancy. Besides, prenatal treatment with dexamethasone in rats at $0.2 \mathrm{mg} / \mathrm{kg} \cdot \mathrm{d}$ in this study is comparable with that used in humans at $0.03 \mathrm{mg} / \mathrm{kg} \cdot \mathrm{d}$. And the standard dose of dexamethasone in clinical settings during pregnancy is $0.05-0.2 \mathrm{mg} / \mathrm{kg} \cdot \mathrm{d}$ (Moisiadis et al. , 2014b). Therefore, we subcutaneously administrated $0.2 \mathrm{mg} / \mathrm{kg} \cdot \mathrm{d}$ dexamethasone on GD9-20 to establish the PDE rat model based on the clinical treatment status and the characteristics of adrenal development.

Glucocorticoids are involved in regulating the biosynthesis and metabolism of sugar, fat, and protein in the body, which play a vital role in the occurrence and development of various chronic diseases (Goodwin et al. , 2012). Longitudinal studies reported that repeated antenatal dexamethasone treatment could cause adverse effects on the growth and development of offspring (Frenchet al. , 1999), such as cognitive and behavioral disorders (French et al. , 2004). Recently, we confirmed multi-organ developmental abnormalities in offspring and susceptibilities to various diseases after birth, such as osteoporosis (Xiao et al. , 2018), neurobehavioral disorders (Huang et al. , 2019), glomerular sclerosis (Liet al. , 2019) in the PDE rat model. In this study, we used the same PDE rat model and found that the adrenal steroidogenic function persistently reduced in the offspring. These findings have significance to understand the occurrence and development of various chronic diseases in adult PDE offspring.

4.2 The low level of maternally derived glucocorticoid exposure in the PDE fetal rats and its maternal-fetal interaction mechanism

In humans, the weight and size of the maternal adrenal gland don't change significantly during pregnancy. 
However, the level of free glucocorticoid in maternal blood is still increased by 2-4 times in the third trimester compared with pre-pregnancy(Nolten et al. , 1981; Rainey et al. , 2004). This indicates that the glucocorticoid synthesis function of the maternal adrenal is enhanced during pregnancy. The development of the fetal adrenal gland is a unique process. Although the fetal adrenal gland can synthesize a small amount of glucocorticoid through progesterone (Macnaughton et al. , 1977), thede novo glucocorticoid synthesis pathway in the fetal adrenal gland was established in the third trimester (Mesiano et al. , 1997). Therefore, the amount of glucocorticoid secreted by the fetal adrenal gland is relatively less than that of the mother, and the fetal blood glucocorticoid in the intrauterine period is mainly derived from maternal blood.

In vivo, we found dexamethasone directly inhibited the maternal adrenal steroidogenesis rather than inhibiting the HPA axis. Further, the serum corticosterone levels were also decreased significantly in the PDE fetal rats. It is known that the maternal glucocorticoid levels during pregnancy are 5-10 times higher than that of the fetus, and the concentration gradient is maintained by the placental glucocorticoid barrier, including Pglycoprotein and 11ß-HSDs system (Zhu et al. , 2019). In this study, we observed PDE opened the placental glucocorticoid barrier. Moreover, we also confirmed that there is a positive correlation between maternal and fetal serum corticosterone levels. In summary, we demonstrated the low exposure to maternal glucocorticoid in the PDE fetal rats was mainly due to the decrease of maternal serum glucocorticoid level and the opening of the placental glucocorticoid barrier, indicating the presence of a maternal-fetal interaction mechanism.

\subsection{The positive programming of GC-IGF1 axis mediated thegender difference in adrenal function of the PDE offspring after birth}

There is a common phenomenon of maternal glucocorticoid overexposure induced by the prenatal adverse environment, such as xenobiotics exposure (Morales-Rubio et al. , 2019), hypoxia (Cuffe et al. , 2014), and stress (Buss et al. , 2012). Our previous research found that the negative regulation change of the GCIGF1 axis was involved in the adrenal developmental programming and homeostasis alterations in offspring with high maternally derived glucocorticoid exposure (Huang et al. , 2015; He et al. , 2019). However, in this study, the serum corticosterone levels decreased in the PDE male offspring, accompanied by the persistent decrease in the H3K27ac level of the IGF1 promoter region, IGF1 signaling pathway and adrenal steroidogenesis. It should be noted that chronic stress significantly increased the serum corticosterone level in adult offspring of the control group, while the above indicators decreased. The serum corticosterone levels were markedly increased in the PDE adult offspring with chronic stress; meanwhile, the above-accompanied indicators were significantly enhanced. In vitro,the lower physiological concentrations of cortisol decreased the IGF1 H3K27ac and expression levels as well as intracellular cortisol production, and the over-expression of GR $\alpha$ could reverse these inhibitory effects of cortisol. In conclusion, PDE-induced maternal glucocorticoid low-exposure caused adrenal insufficiency of the male offspring ratsvia "positive programming" of the GCIGF1 axis.

Exposure to the same environment during pregnancy has different effects on the growth and development in individuals and the occurrence time, course, and outcome of chronic diseases in the offspring of different genders (van Abeelen et al. , 2011; Waddell et al. , 2012; Schaafsma et al. , 2017). Clinic and animal studies reported that the gender difference in catch-up growth patterns is related to the differences in sex hormones in individuals (Perry et al. , 2008). Accumulating evidence has shown that IGF1 plays a vital role in the catchup growth of mammals, and estrogen can promote IGF1 expression through estrogen receptors (Hewittet al. , 2019). This study also sought to determine whether the adrenal development in PDE female offspring is different from that of the males and whether it is also regulated by positive programming of the GCIGF1 axis. In this study, we found the serum corticosterone level of the PDE male offspring decreased after birth, accompanied by continuous inhibition of adrenal IGF1 signaling pathway and steroidogenic function. However, the serum corticosterone level of the PDE females increased after birth (Fig S2), accompanied by continuous enhancement of the above indicators. This suggested that positive programming of the GC-IGF1 axis is also involved in the PDE female adrenal development, and the enhanced female adrenal steroidogenic function after birth might be related to the high level of estrogen to promote IGF1 expression via estrogen receptor. 


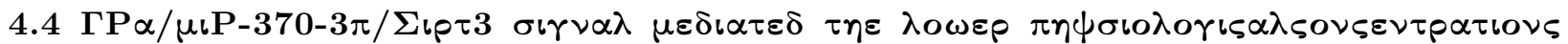

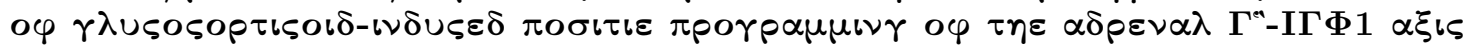

In this study, the levels of H3K27ac of the adrenal IGF1 promoter region decreased continuously in the PDE offspring rats. Further, we found that the expression of adrenal Sirt3 significantly increased in the PDE fetal rats (Fig S3). Mitochondria are the main organelles for adrenal steroidogenesis, and Sirt3 is the main histone deacetylase in mitochondria (Onyango et al. , 2002). We observed that the adrenal Sirt3 expression markedly increased in the PDE offspring rats, while its expression relatively decreased with chronic stress in adulthood. Studies have reported that Sirt3 is targeted by miRNAs (Zhang et al. , 2018). We found that the adrenal miR-370-3p expression reduced in the PDE fetal rats by whole-genome sequence analysis. Meanwhile, miRWalk bioinformatics prediction revealed that the 3'UTR of Sirt3 mRNA had a miR-370$3 \mathrm{p}$ target site. In vivo, the adrenal miR-370-3p expression significantly reduced in the PDE offspring, while its expression relatively increased with chronic stress in adulthood.In vitro, the low physiological concentrations of cortisol concentration-dependently inhibited the miR-370-3p expression and promoted Sirt3 expression. Further, Sirt3 knockdown or miR-370-3p mimics significantly reversed the inhibitory effect of low physiological concentration of cortisol on the H3K27ac and expression levels of IGF1, and miR-370-3p mimics also reversed its promotive effect on Sirt3 expression. Additionally, the luciferase reporter analysis showed that miR-370-3p could directly target Sirt3. It indicated the low physiological concentrations of glucocorticoid downregulated miR-370-3p to target up-regulation of Sirt3, thereby reducing the H3K27ac and expression levels of IGF1 in NCI-H295R cells.

Glucocorticoids mainly exert biological effects through GR, and GR can regulate the expression of noncoding RNA, including miRNA (Tessel et al. , 2010). In this study, we found that the increased expression of fetal adrenal GRain utero was related to the fetal blood dexamethasone exposure, while the adrenal GR $\alpha$ expression alterations after birth was consistent with the serum corticosterone concentration. Moreover, in vitro,the low physiological concentrations of cortisol inhibited the expression of GR $\alpha$ and miR-370-3p, while over-expression of GR $\alpha$ reversed the inhibitory effect of cortisol on miR-370-3p expression. Above all, it indicated the GR $\alpha / \mathrm{miR}-370-3 \mathrm{p} / \mathrm{Sirt} 3$ signal mediated the low physiological concentrations of endogenous glucocorticoids on the positive programming of the adrenal GC-IGF1 axis (Fig 8).

In addition, we were interested in whether the suppression of dexamethasone on adrenal IGF1 expression could last until after birth. We found that the H3K9ac and H3K27ac levels of the adrenal IGF1 promoter region decreased in the PDE fetal rats, but only the H3K27ac level decreased after birth. In vitro, dexamethasone significantly reduced the H3K9ac level of the IGF1 promoter region. It suggested dexamethasone could inhibit adrenal IGF1 expression by reducing the H3K9ac level of the IGF1 promoter region in utero, but this effect could not continue after birth (without dexamethasone exposure).

\section{Conclusions}

This study confirmed that PDE inhibited maternal adrenal function and opened placental glucocorticoid barrier, leading to a low exposure of maternally derived glucocorticoid in the fetal rats. The low level of intrauterine endogenous glucocorticoids could cause adrenal insufficiency in adult male offspring rats, which is mediated by the GR $\alpha /$ miR-370-3p/Sirt3 signal through epigenetic positive programming of the GC-IGF1 axis. However, the inhibitory effect of dexamethasone on adrenal function can't last after birth. It remains to think the causes of gender differences in adrenal function of PDE offspring after birth. This study for the first time systematically confirmed that synthetic glucocorticoids (e.g., dexamethasone) could alter the adrenal development programming and homeostasis in offspring by inhibiting the maternal adrenal function, and it provides an important theoretical and experimental evidence for the comprehensive explanation of maternal adrenal influence on the offspring development and the disease susceptibility.

\section{Acknowledgments}

This work was supported by grants from the National Natural Science Foundation of China (No. 81673524), the National Key Research and Development Program of China (No. 2017YFC1001300), the Major Technological Innovation Projects of Hubei Province (No. 2019ACA140), and Medical Science Advancement 
Program (Basic Medical Sciences) of Wuhan University (No. TFJC2018001).

\section{Author contributions}

H. Wang conceived the study; H. Wang and Y. Chen participated in the study design. Y. Chen, X. Xia, G. Chen, and J. Cao performed the experiments and acquired the data. Y. Chen wrote the manuscript; H. Wang revised the manuscript; All authors read and approved the final manuscript.

\section{Conflict of interests}

All of the authors state that they have no conflicts of interest.

\section{Declaration of transparency and scientific rigour}

This Declaration acknowledges that this paper adheres to the principles for transparent reporting and scientific rigour of preclinical research as stated in the BJP guidelines for Design \& Analysis, Immunoblotting and Immunochemistry and Animal Experimentation, and as recommended by funding agencies, publishers, and other organizations engaged with supporting research.

\section{References:}

Asztalos EV, Murphy KE, Hannah ME, Willan AR, Matthews SG, Ohlsson A, et al. (2010). Multiple courses of antenatal corticosteroids for preterm birth study: 2-year outcomes. Pediatrics 126 (5): e1045-1055.

Buss C, Entringer S, Wadhwa PD (2012). Fetal programming of brain development: Intrauterine stress and susceptibility to psychopathology. Science Signaling5 (245): pt7-pt7.

Clayton SA, Jones SW, Kurowska-Stolarska M, Clark AR (2018). The role of microRNAs in glucocorticoid action. J. Biol. Chem. 293 (6):1865-1874.

Crowther CA, McKinlay CJD, Middleton P, Harding JE (2015). Repeat doses of prenatal corticosteroids for women at risk of preterm birth for improving neonatal health outcomes. Cochrane Database Syst Rev. (7): 215.

Cuffe JS, Walton SL, Singh RR, Spiers JG, Bielefeldt-Ohmann H, Wilkinson L, et al. (2014). Mid- to late term hypoxia in the mouse alters placental morphology, glucocorticoid regulatory pathways and nutrient transporters in a sex-specific manner.J. Physiol. 592 (14): 3127-3141.

Elfayomy AK, Almasry SM (2014). Effects of a single course versus repeated courses of antenatal corticosteroids on fetal growth, placental morphometry and the differential regulation of vascular endothelial growth factor. J. Obstet. Gynaecol. Res. 40 (11): 2135-2145.

Fleming TP, Watkins AJ, Velazquez MA, Mathers JC, Prentice AM, Stephenson J, et al. (2018). Origins of lifetime health around the time of conception: causes and consequences.Lancet 391 (10132): 1842-1852.

Fowden AL (2003). The insulin-like growth factors and feto-placental growth. Placenta24 (8-9): 803-812.

French N, Hagan R, Evans S, Mullan A, Newnham J (2004). Repeated antenatal corticosteroids: Effects on cerebral palsy and childhood behavior. Am. J. Obstet. Gynecol.190: 588-595.

French NP, Hagan R, Evans SF, Godfrey M, Newnham JP (1999). Repeated antenatal corticosteroids: Size at birth and subsequent development. Am. J. Obstet. Gynecol.180 (1): 114-121.

Gao Y, She R, Wang Q, Li Y, Zhang H (2018). Up-regulation of miR-299 suppressed the invasion and migration of HTR-8/SVneo trophoblast cells partly via targeting HDAC2 in pre-eclampsia. Biomedicine $\mathscr{E}$ pharmacotherapy $=$ Biomedecine 83 pharmacotherapie 97: 1222-1228.

Gilstrap LC, Hightower ES, Clewell WH, D'Alton ME, Damon VG, Escobedo MB, et al. (2001). Antenatal corticosteroids revisited: Repeat courses - National Institutes of Health consensus development conference statement, August 17-18, 2000. Obstetrics and gynecology 98 (1): 144-150. 
Goodwin JE, Geller DS (2012). Glucocorticoid-induced hypertension. Pediatr. Nephrol.27 (7): 1059-1066.

Han VK, Lund PK, Lee DC, D'Ercole AJ (1988). Expression of somatomedin/insulin-like growth factor messenger ribonucleic acids in the human fetus: identification, characterization, and tissue distribution. The Journal of clinical endocrinology and metabolism 66 (2): 422-429.

Hatano O, Takakusu A, Nomura M, Morohashi K (1996). Identical origin of adrenal cortex and gonad revealed by expression profiles of Ad4BP/SF-1. Genes Cells 1 (7): 663-671.

He Z, Zhang J, Huang H, Yuan C, Zhu C, Magdalou J, et al. (2019). Glucocorticoid-activation system mediated glucocorticoid-insulin-like growth factor 1 (GC-IGF1) axis programming alteration of adrenal dysfunction induced by prenatal caffeine exposure. Toxicol. Lett. 302: 7-17.

Hewitt SC, Lierz SL, Garcia M, Hamilton KJ, Gruzdev A, Grimm SA, et al. (2019). A distal super enhancer mediates estrogen-dependent mouse uterine-specific gene transcription of Igf1 (insulin-like growth factor 1). J. Biol. Chem. 294 (25): 9746-9759.

Huang H, He Z, Zhu C, Liu L, Kou H, Shen L, et al. (2015). Prenatal ethanol exposure-induced adrenal developmental abnormality of male offspring rats and its possible intrauterine programming mechanisms. Toxicol Appl Pharmacol288 (1): 84-94.

Huang S, Dong W, Jiao Z, Liu J, Li K, Wang H, et al. (2019). Prenatal dexamethasone exposure induced alterations in neurobehavior and hippocampal glutamatergic system balance in female rat offspring. Toxicological sciences : an official journal of the Society of Toxicology : kfz163.

Li B, Zhu Y, Chen H, Gao H, He H, Zuo N, et al. (2019). Decreased H3K9ac level of AT2R mediates the developmental origin of glomerulosclerosis induced by prenatal dexamethasone exposure in male offspring rats. Toxicology411: 32-42.

Liu M, Chen B, Pei L, Zhang Q, Zou Y, Xiao H, et al. (2018). Decreased H3K9ac level of StAR mediated testicular dysplasia induced by prenatal dexamethasone exposure in male offspring rats. Toxicology 408: $1-10$.

Macnaughton MC, Taylor T, McNally EM, Coutts JR (1977). The effect of synthetic ACTH on the metabolism of [4-14C]-progesterone by the previable human fetus. J. Steroid Biochem. 8 (5): 499-504.

Mericq V, Martinez-Aguayo A, Uauy R, Iniguez G, Van der Steen M, Hokken-Koelega A (2017). Long-term metabolic risk among children born premature or small for gestational age.Nature Reviews Endocrinology 13 (1): 50-62.

Mesiano S, Jaffe RB (1997). Developmental and functional biology of the primate fetal adrenal cortex. Endocr. Rev. 18 (3): 378-403.

Mesiano S, Mellon SH, Jaffe RB (1993). Mitogenic action, regulation, and localization of insulin-like growth factors in the human fetal adrenal gland. J. Clin. Endocrinol. Metab. 76 (4): 968-976.

Moisiadis VG, Matthews SG (2014a). Glucocorticoids and fetal programming part 1: Outcomes. Nat. Rev. Endocrinol. 10 (7): 391-402.

Moisiadis VG, Matthews SG (2014b). Glucocorticoids and fetal programming part 2: Mechanisms. Nat. Rev. Endocrinol. 10 (7): 403-411.

Morales-Rubio RA, Alvarado-Cruz I, Manzano-Leon N, Andrade-Oliva MD, Uribe-Ramirez M, QuintanillaVega B, et al. (2019). In utero exposure to ultrafine particles promotes placental stress-induced programming of renin-angiotensin system-related elements in the offspring results in altered blood pressure in adult mice. 16 (1): 7 .

Murphy KE, Hannah ME, Willan AR, Hewson SA, Ohlsson A, Kelly EN, et al. (2008). Multiple courses of antenatal corticosteroids for preterm birth (MACS): a randomised controlled trial. Lancet $\mathbf{3 7 2}$ (9656): 
2143-2151.

Nolten WE, Rueckert PA (1981). Elevated free cortisol index in pregnancy: possible regulatory mechanisms. Am. J. Obstet. Gynecol. 139 (4):492-498.

Onyango P, Celic I, McCaffery JM, Boeke JD, Feinberg AP (2002). SIRT3, a human SIR2 homologue, is an NAD-dependent deacetylase localized to mitochondria. Proc. Natl. Acad. Sci. U. S. A. 99 (21): 13653-13658.

Perry RJ, Farquharson C, Ahmed SF (2008). The role of sex steroids in controlling pubertal growth. Clin. Endocrinol. (Oxf.) 68 (1): 4-15.

Porto AMF, Coutinho IC, Correia JB, Amorim MMR (2011). Effectiveness of antenatal corticosteroids in reducing respiratory disorders in late preterm infants: randomised clinical trial. BMJ-British Medical Journal 342: 7.

Quinlivan JA, Evans SF, Dunlop SA, Beazley LD, Newnham JP (1998). Use of corticosteroids by Australian obstetricians-a survey of clinical practice. Aust. N. Z. J. Obstet. Gynaecol. 38 (1): 1-7.

Raha D, Nehar S, Paswan B, Rebuffat P, Neri G, Naskar R, et al. (2007). IGF-I enhances cortisol secretion from guinea-pig adrenal gland: in vivo and in vitro study.Int. J. Mol. Med. 20 (1): 91-95.

Rainey WE, Rehman KS, Carr BR (2004). Fetal and maternal adrenals in human pregnancy. Obstet. Gynecol. Clin. North Am. 31 (4): 817-835, x.

Ruy CA, van der Voor B, Lafeber HN, van de Lagemaat MV, Rotteveel J, Finken MJJ (2017). Birth weight and postnatal growth in preterm born children are associated with cortisol in early infancy, but not at age 8 years.Psychoneuroendocrinology 82: 75-82.

Schaafsma SM, Gagnidze K, Reyes A, Norstedt N, Mansson K, Francis K, et al. (2017). Sex-specific gene-environment interactions underlying ASD-like behaviors. Proc. Natl. Acad. Sci. U. S. A. 114 (6): 1383-1388.

Sirianni R, Chimento A, Malivindi R, Mazzitelli I, Ando S, Pezzi V (2007). Insulin-like growth factor-I, regulating aromatase expression through steroidogenic factor 1, supports estrogen-dependent tumor Leydig cell proliferation. Cancer Res.67 (17): 8368-8377.

Srivastava T, Merchant RH, Ambadekar MC (1994). Cord blood cortisol levels and respiratory distress syndrome.Indian Pediatr. 31 (8): 923-928.

Tessel MA, Krett NL, Rosen ST (2010). Steroid receptor and microRNA regulation in cancer. Current opinion in oncology 22 (6): 592-597.

van Abeelen AF, de Rooij SR, Osmond C, Painter RC, Veenendaal MV, Bossuyt PM, et al. (2011). The sex-specific effects of famine on the association between placental size and later hypertension. Placenta 32 (9):694-698.

Vogel JP, Souza JP, Gulmezoglu AM, Mori R, Lumbiganon P, Qureshi Z, et al. (2014). Use of antenatal corticosteroids and tocolytic drugs in preterm births in 29 countries: an analysis of the WHO Multicountry Survey on Maternal and Newborn Health. Lancet 384 (9957): 1869-1877.

Waddell J, McCarthy MM (2012). Sexual differentiation of the brain and ADHD: what is a sex difference in prevalence telling us? Current topics in behavioral neurosciences9: 341-360.

Xiao H, Wen Y, Pan Z, Shangguan Y, Qin J, Tan Y, et al. (2018). Increased H3K27ac level of ACE mediates the intergenerational effect of low peak bone mass induced by prenatal dexamethasone exposure in male offspring rats. Cell Death Dis. 9 (6): 638.

Xing T, Zhu J, Xian J, Li A, Wang X, Wang W, et al. (2019). miRNA-548ah promotes the replication and expression of hepatitis B virus by targeting histone deacetylase 4.Life Sci 219: 199-208. 
Xu D, Chen M, Pan XL, Xia LP, Wang H (2011). Dexamethasone induces fetal developmental toxicity through affecting the placental glucocorticoid barrier and depressing fetal adrenal function. Environ. Toxicol. Pharmacol.32 (3): 356-363.

Zhang X, Ji R, Liao X, Castillero E, Kennel PJ, Brunjes DL, et al. (2018). MicroRNA-195 regulates metabolism in failing myocardium via alterations in sirtuin 3 expression and mitochondrial protein acetylation. Circulation 137 (19): 2052-2067.

Zhu P, Wang W, Zuo R, Sun K (2019). Mechanisms for establishment of the placental glucocorticoid barrier, a guard for life. 76 (1): 13-26.

Figure legends

Fig 1. Alterations in the maternal adrenal and placental functions, and the level of CORT in fetal male rats with PDE.(A) Maternal serum CORT concentration; (B) Maternal serum ACTH concentration; (C) The mRNA expression of maternal adrenal steroidogenic genes; (D) Fetal serum CORT con-

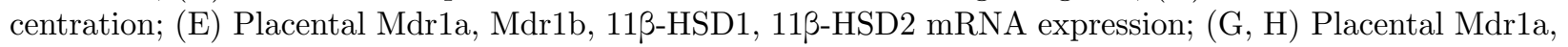

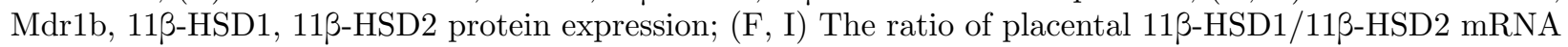
and protein expression; $(\mathrm{J})$ Correlations between maternal serum CORT concentration and fetal serum CORT concentration. $\mathrm{n}=8$ placentas or adrenals for $\mathrm{qRT}-\mathrm{PCR}$ and $\mathrm{n}=3$ for Western blot in each group. Comparisons between groups were performed using Student's $t$-test or Pearson correlation analysis. Mean \pm S.E.M. $,{ }^{*} P<0.05,{ }^{* *} P<0.01 v s$. respective controls. CORT, corticosterone; PDE, prenatal dexamethasone exposure; ACTH, adrenocorticotropic hormone; Mdr1a, multidrug-resistance protein 1a; 11ß-HSD1, hydroxysteroid 11-beta dehydrogenase 1; StAR, steroidogenic acute regulatory protein; P450scc, cytochrome P450

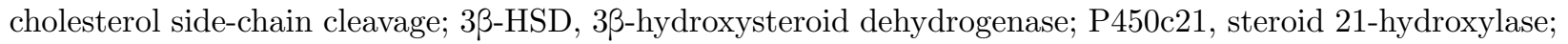
P450c11, steroid 11ß-hydroxylase.

Fig 2. Alterations in serum CORT level, histone acetylation and expression levels of IGF1, adrenal steroidogenesis, and related indicators in fetal male rats with PDE.(A, D, E, H) The mRNA expression of fetal adrenal steroidogenic genes, IGF1 signal pathway, GR $\alpha$ and Sirt3; (B, C) StAR protein expression (yellow represents StAR positive cells, 400×); (F) Clustering of miRNAs differentially expressed in fetal adrenal; (G) Adrenal miR-370-3p expression; (I) Enrichment of H3K9ac, H3K14ac and H3K27ac levels of IGF1 promoter region; preimmune IgG served as the negative control. $\mathrm{n}=8$ adrenals for $\mathrm{qRT}-\mathrm{PCR}$ and $\mathrm{n}=5$ for IHC/ChIP assay in each group. Comparisons between groups were performed using Student's $t$-test Mean \pm S.E.M. ${ }^{*} P<0.05,{ }^{* *} P<0.01$ vs . respective controls. CORT, corticosterone; IGF1, insulin-like growth factor 1; PDE, prenatal dexamethasone exposure; GR $\alpha$, glucocorticoid receptor alpha; H3K9ac, histone 3 lysine 9 acetylation; StAR, steroidogenic acute regulatory protein; P450scc, cy-

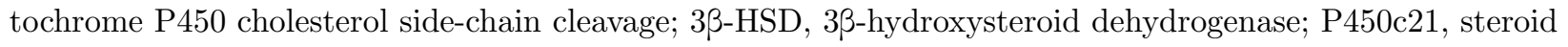
21-hydroxylase; P450c11, steroid 11ß-hydroxylase; IGF1R, IGF1 receptor; Akt1, protein kinase B.

Fig 3. Alterations in serum CORT level, histone acetylation and expression levels of IGF1, adrenal steroidogenesis, and related indicators $s$ in male offspring rats with PDE.(A) Adult serum CORT concentration; (B, D, E, G) Adrenal GR $\alpha$, Sirt3, IGF1 signal pathway, StAR and P450scc mRNA expression; (F) miR-370-3p expression; (C) Adrenal StAR protein expression (yellow represents StAR positive cells, 400×); (H) Enrichment of H3K9ac, H3K14ac and H3K27ac levels of IGF1 promoter region; preimmune IgG served as the negative control. $n=8$ adrenals for qRT-PCR and $n=5$ for IHC/ChIP assay in each group. Comparisons between groups were performed using Student'st -test. Mean \pm S.E.M. ${ }^{*} P$ $<0.05,{ }^{* *} P<0.01$ vs . respective controls. CORT, corticosterone; IGF1, insulin-like growth factor 1; PDE, prenatal dexamethasone exposure; GR $\alpha$, glucocorticoid receptor alpha; StAR, steroidogenic acute regulatory protein; P450scc, cytochrome P450 cholesterol side-chain cleavage; H3K9ac, histone 3 lysine 9 acetylation; IGF1R, IGF1 receptor; Akt1, protein kinase B.

Fig 4. Effects of chronic stress on CORT level, the histone acetylation and expression levels of IGF1, steroidogenesis, and related indicators in male rats with PDE.(A) Serum CORT concentration; (B-D,F) Adrenal GR $\alpha$, Sirt3, IGF1, StAR and P450scc mRNA expression; (E) miR-370-3p expression; 
(G) Enrichment of H3K27ac level of IGF1 promoter region; preimmune IgG served as the negative control. $\mathrm{n}=8$ adrenals for qRT-PCR and $\mathrm{n}=5$ for ChIP assay in each group. Mean \pm S.E.M., ${ }^{*} P<0.05,{ }^{* *} P<0.01$ vs . respective controls. CORT, corticosterone; IGF1, insulin-like growth factor 1; PDE, prenatal dexamethasone exposure; GR $\alpha$, glucocorticoid receptor alpha; StAR, steroidogenic acute regulatory protein; P450scc, cytochrome P450 cholesterol side-chain cleavage; H3K27ac, histone 3 lysine 27 acetylation.

Fig 5. Effects of dexamethasone on the histone acetylation and expression levels of IGF1 and steroidogenesis in NCI-H295R cells.(A) IGF1 mRNA expression; (B, E) StAR mRNA expression; (C) IGF1 and StAR protein expression; (D) Intracellular cortisol concentration; (F) Enrichment of H3K9ac, H3K14ac, H3K27ac levels of IGF1 promoter region; preimmune IgG served as the negative control. $\mathrm{n}=6$ for qRT-PCR from triplicate independent experiments and $n=5$ for Western blot in each group. Mean \pm S.E.M. $,{ }^{*} P<0.05,{ }^{* *} P<0.01$ vs control; ${ }^{\#} P<0.05$, ${ }^{\# \#} P<0.01$ vs $500 \mathrm{nM}$ dexamethasone by one-way ANOVA or Student's $t$-test. IGF1, insulin-like growth factor 1; StAR, steroidogenic acute regulatory protein; H3K9ac, histone 3 lysine 9 acetylation.

Fig 6. Effects of low physiological concentrations of cortisol on the histone acetylation and expression levels of IGF1, steroidogenesis and related indicators in NCI-H295R cells. (A, CE, G) StAR, GR $\alpha$, miR-370-3p, Sirt3 and IGF1 expression; (B) Intracellular cortisol concentration; (G) Enrichment of H3K9ac, H3K14ac, H3K27ac levels of IGF1 promoter region; preimmune IgG served as the negative control; (H) StAR, Sirt3 and IGF1 protein expression. $n=6$ for qRT-PCR from triplicate independent experiments and $n=5$ for Western blot/ChIP assay in each group. Comparisons between groups were performed using Student's $t$-test and ANOVA. Mean \pm S.E.M. ${ }^{*} P<0.05,{ }^{* *} P<0.01 \mathrm{vs}$. respective controls. IGF1, insulin-like growth factor 1; StAR, steroidogenic acute regulatory protein; GR $\alpha$, glucocorticoid receptor alpha; H3K9ac, histone 3 lysine 9 acetylation.

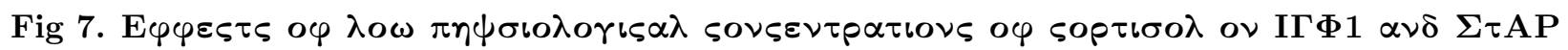

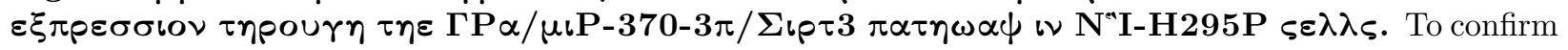
the roles of GR $\alpha$, miR-370-3p, and Sirt3 in the gene regulation of cortisol, we incubated the cells with cortisol $(300 \mathrm{nM})$ in the absence or presence of GR $\alpha$ plasmid, miR-370-3p mimics, Sirt3 siRNA, IGF1 plasmid, respectively, for $24 \mathrm{~h}$ and then harvested. (A) StAR mRNA expression; (B, E, J) Enrichment of H3K27ac level of IGF1 promoter region; preimmune IgG served as the negative control; (C, F, K) IGF1 expression; (D) Sirt3 expression; (G) Sequence of the WT or mutated miR-370-3p binding site within the 3'-UTR of Sirt3 mRNA; (H) The association between miR-370-3p and Sirt3 by luciferase assay; (I) miR-370-3p expression. $\mathrm{n}=6$ for $\mathrm{qRT}-\mathrm{PCR}$ from triplicate independent experiments and $\mathrm{n}=5$ for Western blot/ChIP assay in each group. Mean \pm S.E.M. ${ }^{*} P<0.05,{ }^{* *} P<0.01$ vs control; ${ }^{*} P<0.05$, $\# \# P<0.01$ vs $75 \mathrm{nM}$ cortisol by oneway ANOVA or Student's $t$-test. IGF1, insulin-like growth factor 1; StAR, steroidogenic acute regulatory protein; GR $\alpha$, glucocorticoid receptor alpha; H3K27ac, histone 3 lysine 27 acetylation.

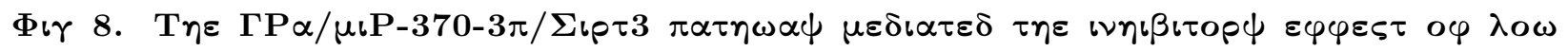

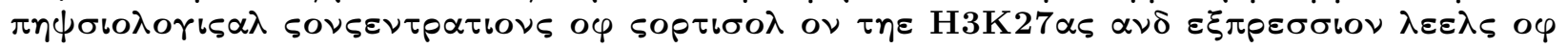
IГ $\mathbf{\Phi} 1$. GR $\alpha$, glucocorticoid receptor alpha; GRE, glucocorticoid receptor element; IGF1, insulin-like growth factor 1; H3K27ac, histone 3 lysine 27 acetylation.

\section{Supplementary information:}

Fig S1. The schedule of animal treatment from gestational day (GD) 0 to postnatal week (PW) 28.

Fig S2. Alterations in serum corticosterone (CORT) level, insulin-like growth factor 1 (IGF1) signal pathway, and adrenal steroidal synthase expression in female adult offspring rats with prenatal dexamethasone exposure.

Fig S3. Alterations in expression of histone acetylases and deacetylases in the fetal adrenal with prenatal dexamethasone exposure (PDE).

Table S1. Oligonucleotide primers for qRT-PCR. 
Table S2. Oligonucleotide primers for ChIP-PCR.
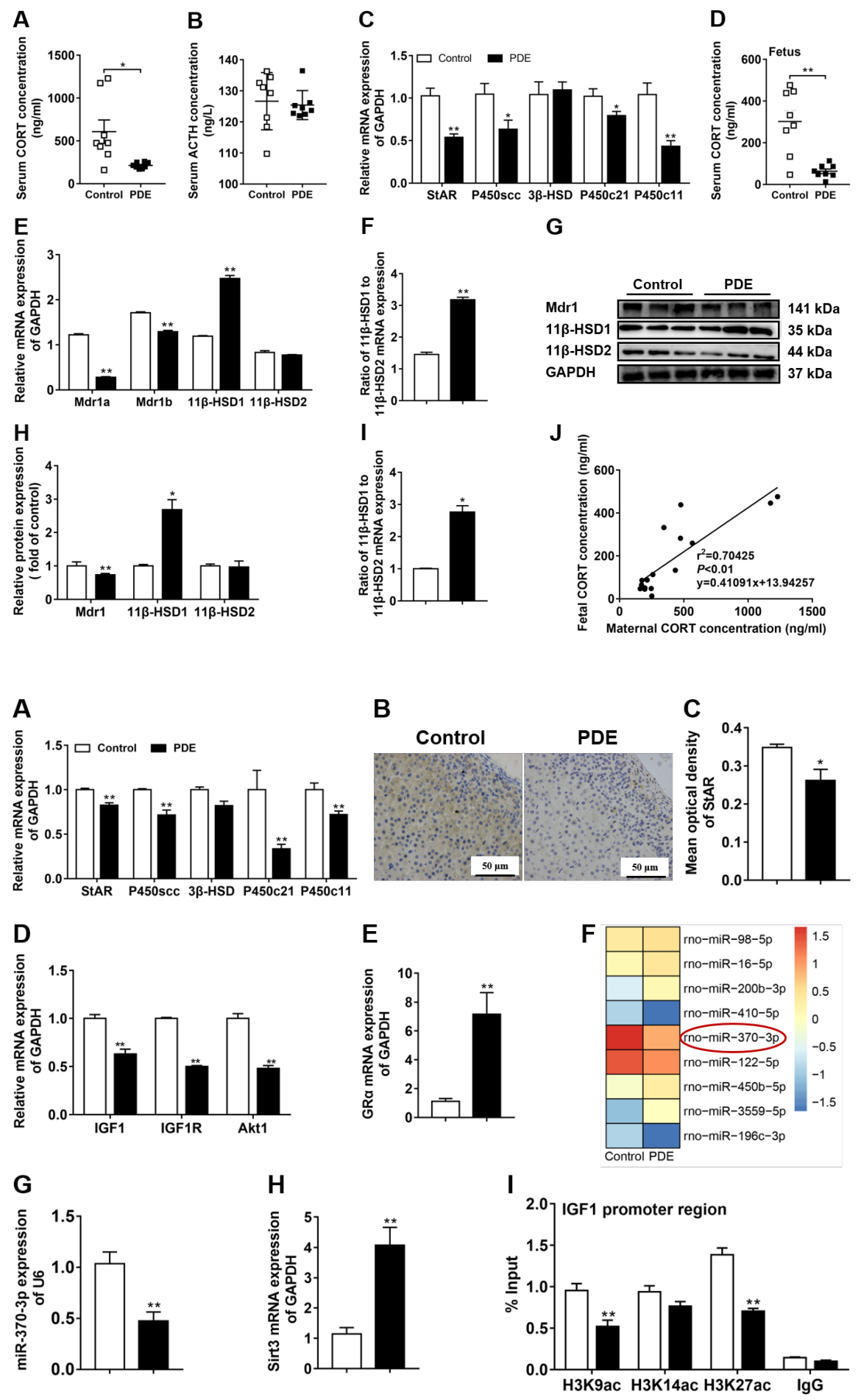

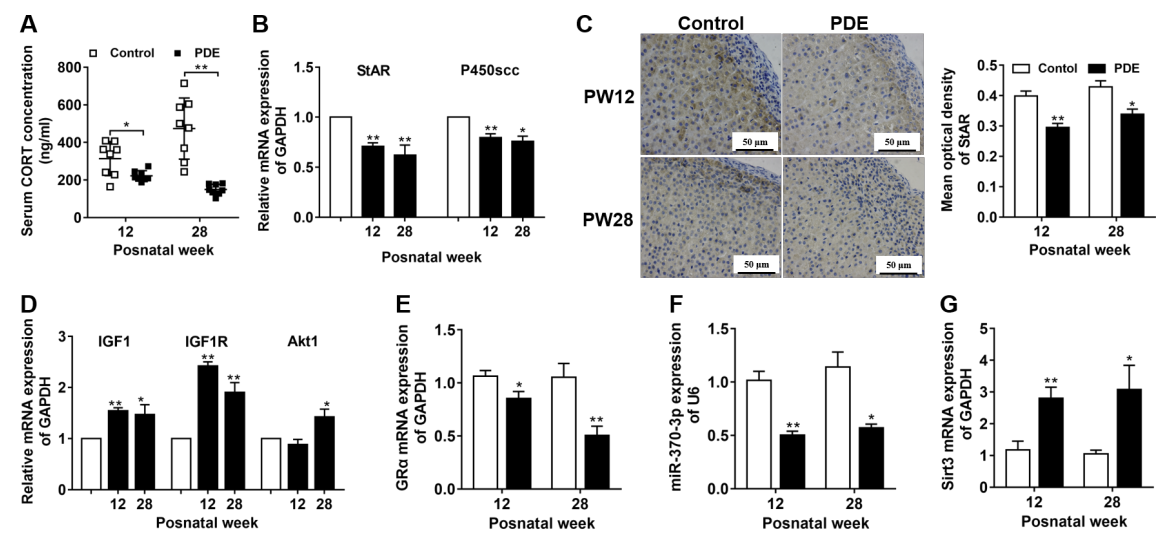

"
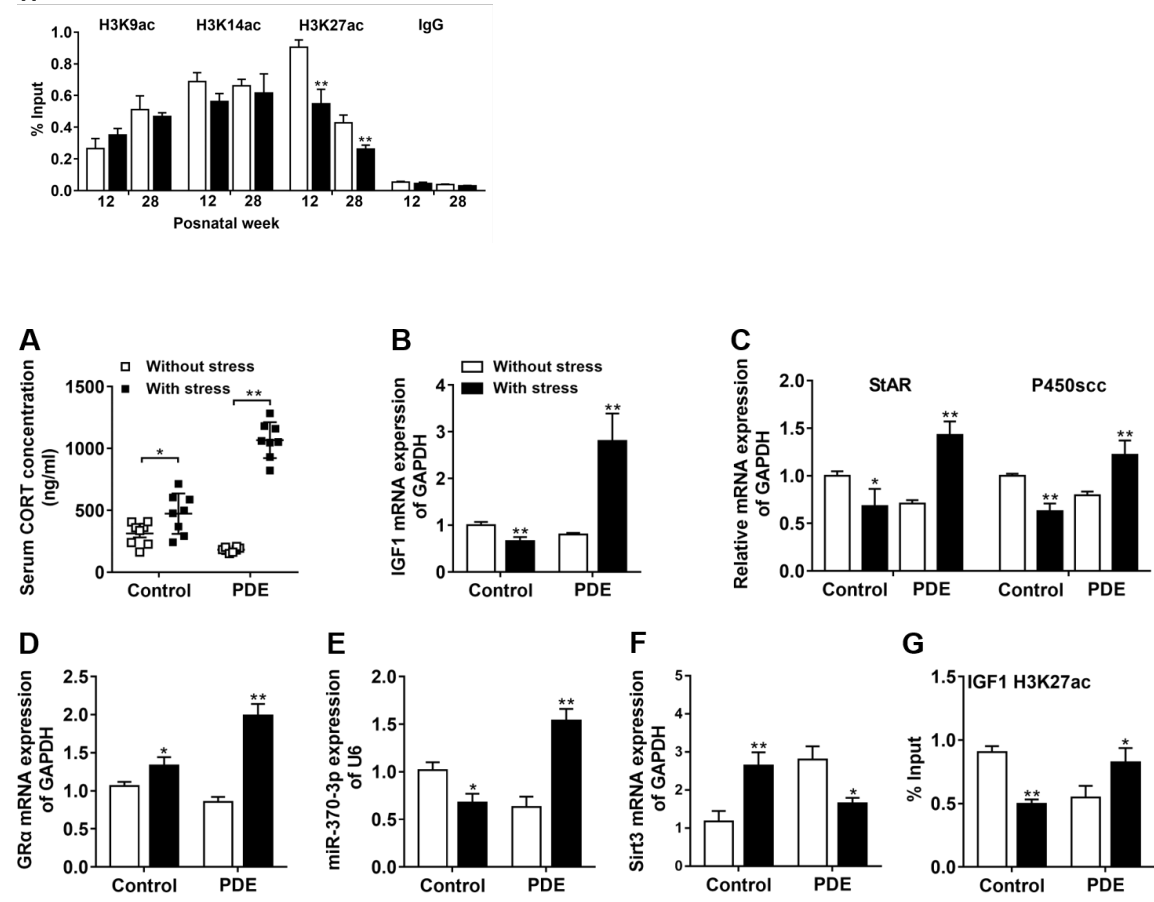
A
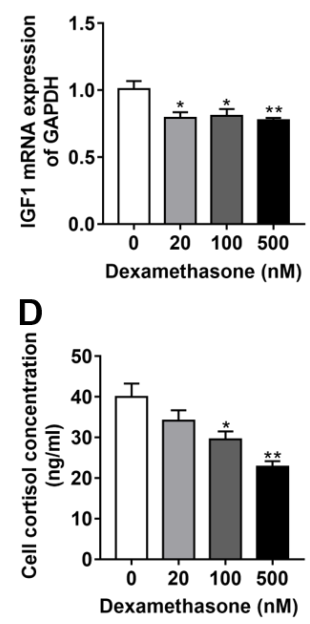

B

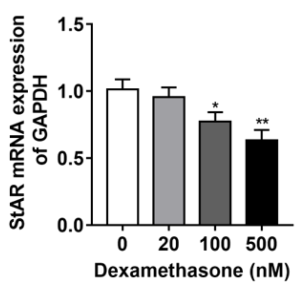

E

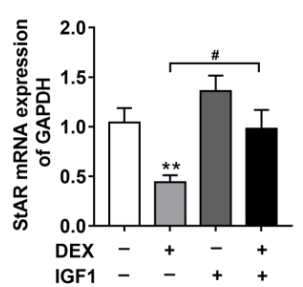

C

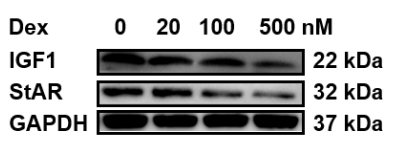

F $\square$ Control $\square$ 500nM Dex

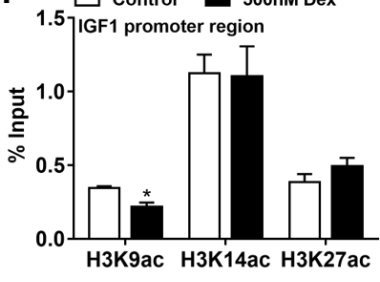

A

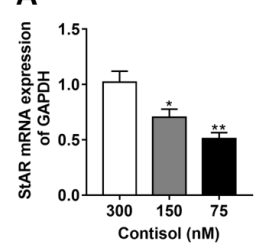

E $F$

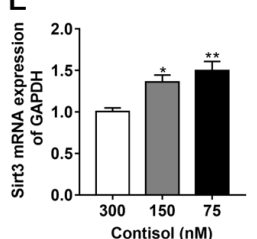

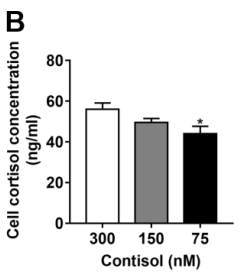

${ }^{3}$ IGF1 promoter region

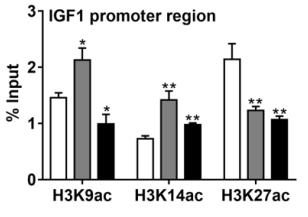

C

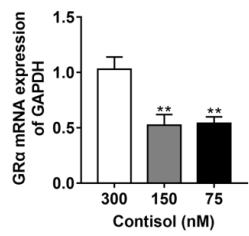

G

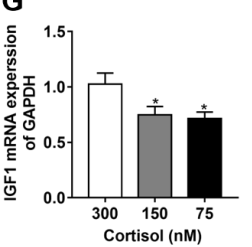

D

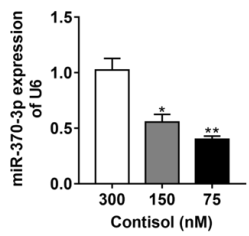

H

Cortisol $300 \quad 150 \quad 75 \mathrm{nM}$

StAR $32 \mathrm{kDa}$

Sirt3 $28 \mathrm{kDa}$

Sirt3 $28 \mathrm{kDa}$

GAPDH $37 \mathrm{kDa}$ 


\section{A}

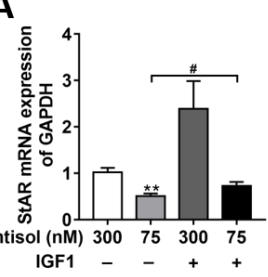

D

Sirt3 $-28 \mathrm{kDa}$

GAPDH $37 \mathrm{kDa}$

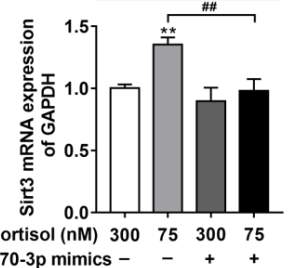

G
I

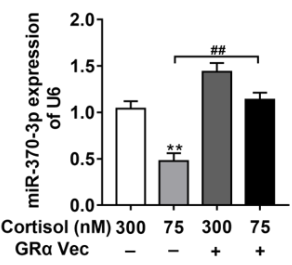

B

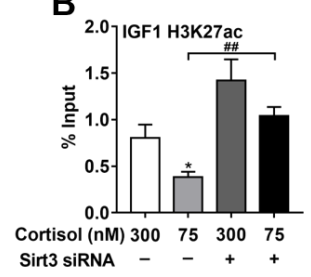

E

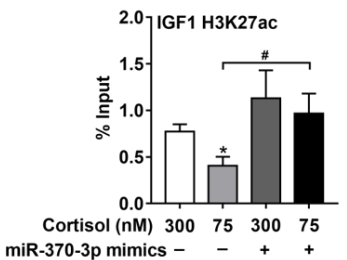
miR-370-3p mimics -

CGACCCC 416

IIIIIII

hsa-miR-370-3p GCCUGCUGGGGUGGAACCUGGU

Sirt3 3'UTR Mut 422 GCTGGGG 416

\section{J}

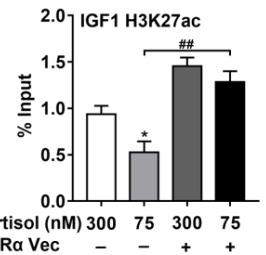

C

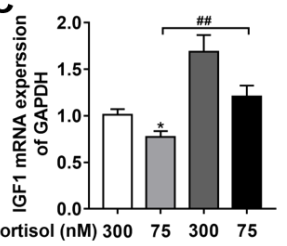

Cortisol (nM) $300 \quad 75 \quad 300 \quad 75$ Sirt3 siRNA

$F$

IGF1 $22 \mathrm{kDa}$
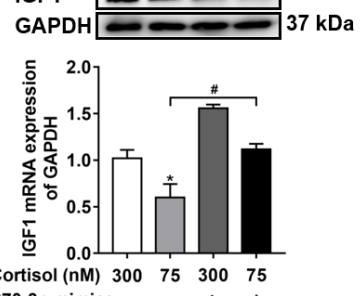

miR-370-3p mimics - -++

$\mathrm{H}$

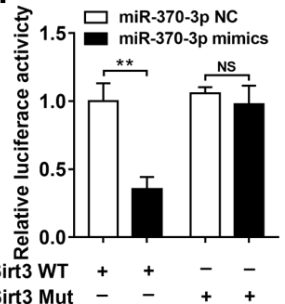

$\mathbf{K}$

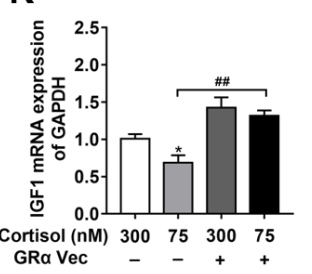




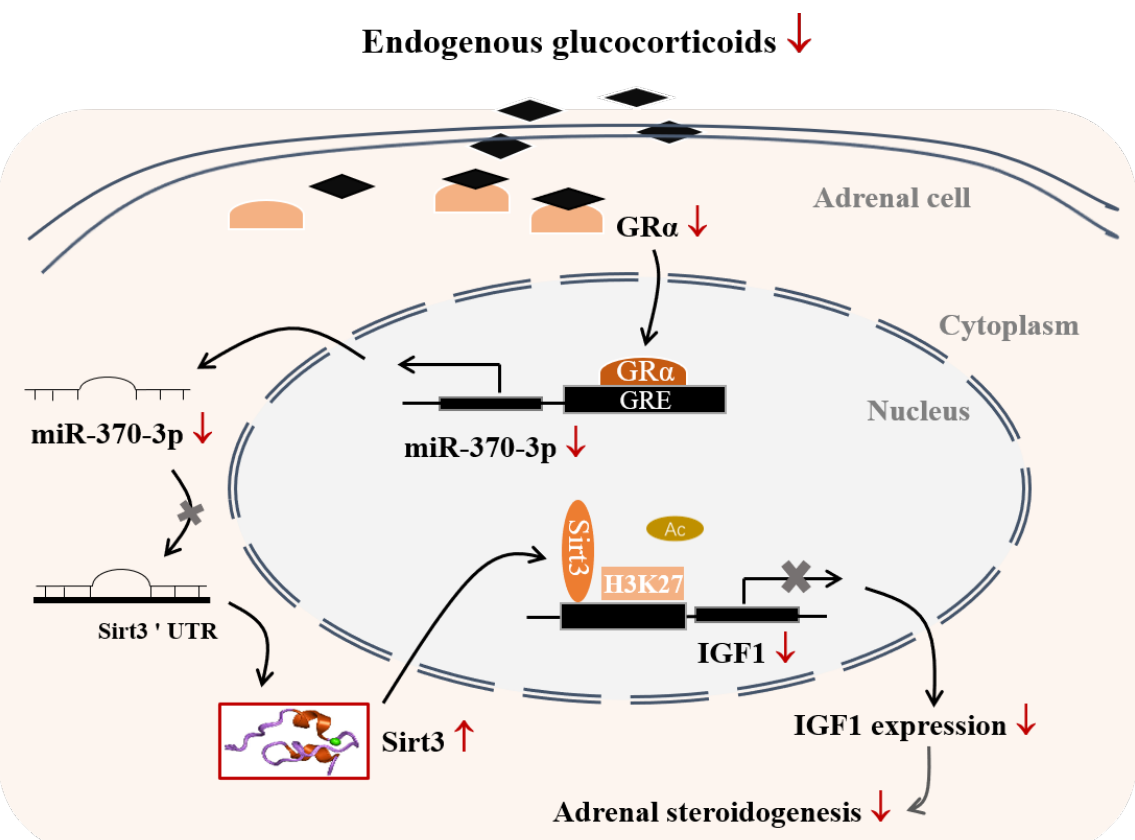

